\title{
Miscelánea
}

\section{Entrevista a María Vizcaíno Gómez}

Facultativo Especialista. Miembro de la Comisión de seguimiento de actuación sanitaria ante la MGF de la

Comunidad Valenciana. Servicio: Ginecología y Obstetricia. Hospital UniversitarioVinalopó, Elche.

Cómo citar esta entrevista en edición digital: Siles, J. \& Solano, C. (2018). Entrevista a María Vizcaíno Gómez.

Cultura de los Cuidados (Edición digital), 22(50). Recuperado de http://dx.doi.org/10.14198/cuid.2018.50.17

Correspondencia: Remitirse al correo electrónico.

Correo electrónico: mvizcaino@vinaloposalud.com

Recibido: 08/03/2018; Aceptado: Entrevista invitada.

\section{ABSTRACT}

In this brief interview with a specialist in gynecology member of the commission of monitoring of Female Genital Mutilation in the Valencian Community, are treated essential aspects of Female Genital Mutilation and especially its incidence in the context of the Valencian Community.

Keywords: Female genital mutilation, gynecology, gender violence.

\section{RESUMEN}

En esta breve entrevista realizada a una especialista en ginecología miembro de la comisión de seguimiento de la Mutilación Genital Femenina en la Comunidad Valenciana, se tratan aspectos esenciales de la Mutilación Genital Femenina y especialmente su incidencia en el contexto de la Comunidad Valenciana.

Palabras clave: Mutilación genital femenina, ginecología, violencia de género.

\section{RESUMO}

Nesta breve entrevista com um especialista em ginecologia membro da comissão de monitorização da Mutilação Genital Feminina na Comunidade Valenciana, são tratados os aspectos essenciais da Mutilação Genital Feminina e especialmente a sua incidência no contexto da Comunidade Valenciana.
Palavras-chave: Mutilação genital feminina, ginecologia, violência de gênero.

- Cuéntanos algo sobre tus principios como persona y como profesional; cuándo y por qué decidiste dedicarte a la medicina?

Resulta muy difícil definirse a uno mismo como persona y como profesional. $\mathrm{Me}$ considero una persona normal, comprometida con mi familia y mi trabajo y que intenta ser perfeccionista en todo aquello que hace.

Realmente no recuerdo el momento exacto en el que decidí dedicarme a la medicina. Es una vocación que no viene determinada en mi caso por la tradición, ya que en mi familia no hay ningún médico ...pero sin embargo, tengo la sensación de que toda la vida he tenido claro que eso era lo que me gustaba, al menos desde que he sido capaz de generar recuerdos. Siempre me ha llamado la atención el cuerpo humano y el estudio de su anatomía y funcionamiento así como el poder ayudar a los demás mejorando con nuestros conocimientos su calidad de vida.

- ¿Dónde realizaste tus estudios..., dónde te formaste como médica y cómo ginecóloga?

Estudié la carrera de medicina en la Universidad de Murcia, cursando un año en La Universitat degli Studi di Firenze con una beca Erasmus. Tras finalizar la carrera y hacer 
el examen MIR, me formé en la especialidad de Ginecología y Obstetricia en el Hospital General Universitario de Elche en el que trabajé primero como residente y posteriormente de facultativa especialista desde 2005 hasta Junio de 2010 , año en el que comencé a trabajar en el Hospital Universitario Vinalopó que es donde actualmente desempeño mi ejercicio profesional.

\section{- ¿Cuándo y dónde te sensibilizaste con el} problema de la MGF...cuándo identificaste

\section{los primeros casos de MGF?}

Ser consciente de que existía dicha práctica lo he sido desde que comencé a dedicarme a la medicina aunque realmente tenía una percepción del tema como algo lejano y minoritario. Sin embargo esa percepción ha cambiado de forma sustancial y mi senbilidad ha aumentado a raiz de comenzar a formar parte de la comisión de seguimiento de actuación sanitaria ante la MGF de la Comunidad Valenciana hace algo más de un año. Muy lejos de mi idea inicial se trata de un problema visible en nuestro entorno no tan raro ni tan lejano y sobre todo, sobre el que podemos influir y debemos hacerlo para ayudar a las mujeres que por desgracia ya han sufrido procedimientos de mutilación genital $\mathrm{y}$ algo fundamental, intervenir activamente para evitar que se siga cometiendo este tipo de violencia de género hoy en día. Debemos proteger a las niñas en riesgo de mutilación y tenemos a nuestra disposición todo lo necesario para hacerlo.

Desde que formamos parte de dicha comisión hemos detectado en nuestro departamento 8 casos de mujeres mutiladas y 5 niñas de su entorno que podrían estar en situación de especial vulnerabilidad $y$ riesgo de mutilación. Los primeros casos se diagnosticaron en Abril de 2017.
- ¿Cómo reaccionan las mujeres cuando se les habla sobre la MGF de la que han sido objeto-sujeto?

La primera reacción suele ser la negación. Cuando se va realizando un abordaje más gradual en diferentes consultas, seles ofrece más información y somos capaces de transmitirles que nuestro objetivo lejos de fiscalizarlas, es ayudarlas y prestarles la atención integral que puedan necesitar ofreciéndoles apoyo para el tratamiento de todas aquellas complicaciones físicas y psicológicas que pudieran haber sido derivadas de su estado de mutilación, por lo general admiten dicha práctica. Explican cómo en sus países es un procedimiento totalmente normalizado y llama la atención que en muchos casos, ni siquiera son conscientes de que algunas de las patologías que sufren o han sufrido a lo largo de su vida puedan estar en relación con la mutilación genital.

- ¿Cómo te ha repercutido adoptar una actitud y posicionamiento contra esta práctica?

Tanto a nivel personal como profesional han sido todo sensaciones y repercusiones positivas. He percibido el apoyo y el interés de todos los compañeros y para mí supone una gran satisfacción poder vehiculizar todas las herramientas que se han puesto a nuestra disposición para detectar precozmente a las mujeres que victimas de esta violencia y prevenir que en el futuro más niñas sean sometidas a este tipo de prácticas.

- ¿Nos podrías decir -en tu opinión- si tus colegas tienen tan clara como tú la necesidad de visibilizar esta práctica en España?

La percepción en mi departamento ha sido que sí que lo tienen claro y desde el primer momento ha habido una gran implicación y 
colaboración para que así sea empezando por el Dr. Cazorla que es mi jefe de servicio y que fue el que pensó en mí para formar parte de este grupo de trabajo, mis compañeros ginecólogos, trabajadores sociales y resto de compañeros de personal sanitario, tanto de atención especializada como de atención primaria. No obstante queda todavía mucho trabajo por hacer. Es necesario seguir dando visibilidad a este problema y seguir transmitiendo y contagiando ese espíritu de colaboración entre todos a otros departamentos para que finalmente se genere una red de trabajo realmente eficaz.

- ¿Existen protocolos para trabajar en equipo en pro de la identificación de esta práctica?

En 2015 se publicó el Protocolo Común de actuación sanitaria ante la MGF por parte del Ministerio de Sanidad, Servicios Sociales e Igualdad. A partir de entonces, se comenzaron a realizar protocolos en varias Comunidades Autónomas. En la Comunidad Valenciana se inició el grupo de trabajo en Febrero de 2016 y finalmente se presentó oficialmente el Protocolo de actuación en Noviembre de 2016 por la Consellería de Sanidad.

En este protocolo quedan reflejados diferentes algoritmos de intervención según los distintos grupos de población y ámbitos sanitarios.

- ¿Qué estrategias desarrollarías para conciencias a tus colegas del problema de la MGF?

Desarrollaría estrategias en primer lugar de difusión del protocolo y de sensibilización frente a esta problemática. En segundo lugar, programaría acciones formativas específicas para todo el personal sanitario y finalmente el objetivo que debemos perseguir es generar un sistema de coordinación con otras administraciones que nos permita llevar a cabo un abordaje multidisciplinar e integral de la MGF. 